Homology, Homotopy and Applications, vol.3(3), 2001, pp.453-466

\title{
EXACT COMPLETION AND REPRESENTATIONS IN ABELIAN CATEGORIES
}

\author{
J. ROSICKÝ AND E.M. VITALE \\ (communicated by Cristina Pedicchio)
}

\begin{abstract}
When the exact completion of a category with weak finite limits is a Mal'cev category, it is possible to combine the universal property of the exact completion and the universal property of the coequalizer completion. We use this fact to explain Freyd's representation theorems in abelian and Frobenius categories.
\end{abstract}

\section{Introduction}

In the first of his contributions to the La Jolla proceedings [7], P. Freyd shows that a small abelian category with enough projectives is the reflection of any of its projective covers (which are preadditive categories) in the 2-category of small abelian categories. Moreover, Freyd characterizes those small preadditive categories which occur as projective covers of small abelian categories.

There is a strong analogy between Freyd's results and the properties of the exact completion established in [6]. The aim of this note is to make precise this analogy : we show that Freyd's results follow using at the same time the exact completion of a category with weak finite limits and the reflexive coequalizer completion of a category $[\mathbf{1 3}, \mathbf{3}]$. It is remarkable that the basic ingredient to combine the two completions is that abelian categories are Mal'cev categories : if the exact completion is a Mal'cev category, then it coincides with the reflexive coequalizer completion.

To complete our analysis, in the last section we consider the fact, proved in [8], that an additive functor from a projective-injective cover of a Frobenius category admits three different extensions to the whole category. This leads us to compare the exact completion with a third kind of completion, the pre-regular completion. We establish a necessary and sufficient condition on the base category for its exact and pre-regular completions to be equivalent. In the additive case, this condition is equivalent to be a projective-injective cover of a Frobenius category, and the universal property of a Frobenius category follows from the equivalence of the three quoted completions. Another corollary of our analysis is that the pre-regular completion of a triangulated category $\mathbb{T}$ is the universal homological functor on $\mathbb{T}$. This

First Author supported by the Grant Agency of the Czech Republic under the grant No. 201/99/0310.

Received November 30, 2000; published on September 27, 2001.

2000 Mathematics Subject Classification: 18A35, 18B15, 18E10, 18G05.

Key words and phrases: completions, Mal'cev categories, abelian categories, Frobenius categories. (C) 2001, J. Rosický and E.M. Vitale. Permission to copy for private use granted. 
fact, first proved in $[8]$ when $\mathbb{T}$ is the stable homotopy category of spaces, has been generalized to arbitrary triangulated categories by M. Grandis [9] and A. Neeman $[\mathbf{1 1}]$.

A warning : the composition of two arrows $X \stackrel{f}{\longrightarrow} Y \stackrel{g}{\longrightarrow} Z$ is written $f \cdot g$.

Aknowledgment : We warmly thank the editor and the referee for their help about the correct formulation and proof of Lemma 8.

\section{Review on free completions}

In this section we recall construction and universal property of the various completions we need.

Recall that a regular category is a category with finite limits and (regular epi, mono) factorizations, in which regular epis are pullback stable. A category is exact if it is regular and moreover equivalence relations are effective (i.e., they have a coequalizer and they are the kernel pairs of their coequalizer) $[\mathbf{1}, \mathbf{2}]$. A functor between regular or exact categories is exact if it preserves finite limits and regular epis. Recall also that a weak limit is defined as a limit, but deleting the uniqueness of the factorization. A functor $F: \mathbb{C} \rightarrow \mathbb{B}$, with $\mathbb{C}$ having weak finite limits and $\mathbb{B}$ exact, is left covering if for each finite diagram $\mathcal{D}$ in $\mathbb{C}$ and for each weak limit $W$ of $\mathcal{D}$, the comparison between $F(W)$ and the limit of $F(\mathcal{D})$ is a regular epi.

In $[6]$ the following results have been proved.

Proposition 1. Let $\mathbb{C}$ be a category with weak finite limits. Then

1) there exists an exact category $\mathbb{C}_{e x}$ and a full and faithful functor

$$
\Gamma: \mathbb{C} \rightarrow \mathbb{C}_{e x}
$$

(the "exact completion" of $\mathbb{C}$ ) such that, for each exact category $\mathbb{B}$, the composition with $\Gamma$ induces an equivalence between the category of exact functors from $\mathbb{C}_{e x}$ to $\mathbb{B}$ and the category of left covering functors from $\mathbb{C}$ to $\mathbb{B}$

$$
\Gamma \cdot-: \operatorname{Ex}\left[\mathbb{C}_{e x}, \mathbb{B}\right] \rightarrow \operatorname{Lco}[\mathbb{C}, \mathbb{B}] ;
$$

2) $\Gamma(\mathbb{C})$ is a projective cover of $\mathbb{C}_{e x}$, i.e. each object of $\Gamma(\mathbb{C})$ is regular projective, and each object of $\mathbb{C}_{e x}$ is a regular quotient of an object of $\Gamma(\mathbb{C})$;

3) if $\mathbb{A}$ is an exact category and $\mathbb{P}$ is a projective cover of $\mathbb{A}$ then $\mathbb{P}$ has weak finite limits, the full inclusion $\mathbb{P} \rightarrow \mathbb{A}$ is a left covering functor and its exact extension $\mathbb{P}_{e x} \rightarrow \mathbb{A}$ is an equivalence.

The exact category $\mathbb{C}_{e x}$ can be described in the following way :

- an object of $\mathbb{C}_{e x}$ is a pseudo-equivalence $X_{1} \underset{x_{1}}{\stackrel{x_{0}}{\longrightarrow}} X_{0}$ in $\mathbb{C}$ (that is, as an equivalence relation, but without requiring that the pair is jointly monic); 
- a premorphism in $\mathbb{C}_{e x}$ is a pair $(f, \bar{f})$ of arrows in $\mathbb{C}$ as in the following diagram

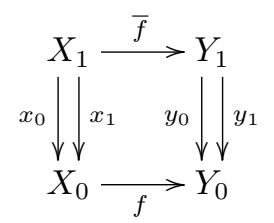

such that $x_{0} \cdot f=\bar{f} \cdot y_{0}$ and $x_{1} \cdot f=\bar{f} \cdot y_{1}$

- a morphism in $\mathbb{C}_{e x}$ is an equivalence class $[f, \bar{f}]$ of premorphisms : we identify two pairs $(f, \bar{f})$ and $(g, \bar{g})$ of premorphisms if there exists $l: X_{0} \rightarrow Y_{1}$ such that $l \cdot y_{0}=f$ and $l \cdot y_{1}=g$;

- composition and identities in $\mathbb{C}_{e x}$, and the full embedding $\Gamma$, are the obvious ones.

Let us now recall from $[\mathbf{1 3}, \mathbf{3}]$ the reflexive coequalizer completion of a category (a reflexive coequalizer is the coequalizer of a reflexive graph).

Proposition 2. Let $\mathbb{C}$ be any category. Then

1) there exists a category $\mathbb{C}_{r c}$ with reflexive coequalizers and a full and faithful functor

$$
\Lambda: \mathbb{C} \rightarrow \mathbb{C}_{r c}
$$

such that, for each category $\mathbb{B}$ with reflexive coequalizers, the composition with $\Lambda$ induces an equivalence between the category of reflexive coequalizer preserving functors from $\mathbb{C}_{r c}$ to $\mathbb{B}$ and the category of functors from $\mathbb{C}$ to $\mathbb{B}$

$$
\Lambda \cdot-: R C\left[\mathbb{C}_{r c}, \mathbb{B}\right] \rightarrow[\mathbb{C}, \mathbb{B}] ;
$$

2) if moreover $\mathbb{C}$ has finite sums, then $\mathbb{C}_{r c}$ has finite colimits and, for each category $\mathbb{B}$ with finite colimits, the composition with $\Lambda$ induces an equivalence between the category of right exact (= finite colimits preserving) functors from $\mathbb{C}_{r c}$ to $\mathbb{B}$ and the category of finite sums preserving functors from $\mathbb{C}$ to $\mathbb{B}$

$$
\Lambda \cdot-: \operatorname{Right}\left[\mathbb{C}_{r c}, \mathbb{B}\right] \rightarrow F S[\mathbb{C}, \mathbb{B}]
$$

The category $\mathbb{C}_{r c}$ can be described as follows :

- an object in $\mathbb{C}_{r c}$ is a reflexive graph $X_{1} \underset{x_{1}}{\stackrel{x_{0}}{\longrightarrow}} X_{0}$ in $\mathbb{C}$

- consider the following diagram in $\mathbb{C}$, with $Y_{1} \underset{y_{1}}{\stackrel{y_{0}}{\longrightarrow}} \geq Y_{0}$ a reflexive graph

$$
\begin{gathered}
Y_{1} \\
V \stackrel{f}{y_{0} \downarrow}{ }_{g} \longrightarrow y_{0}
\end{gathered}
$$

we say that $f$ is preequivalent to $g$ if there exists $h: V \rightarrow Y_{1}$ such that $h \cdot y_{0}=f$ and $h \cdot y_{1}=g$; this is a reflexive relation in the hom-set $\mathbb{C}\left(V, Y_{0}\right)$. We write 
$f \simeq g$ if $f$ and $g$ are in the equivalence relation generated by the above reflexive relation ;

- a premorphism in $\mathbb{C}_{r c}$ is an arrow $f$ of $\mathbb{C}$ as in the diagram

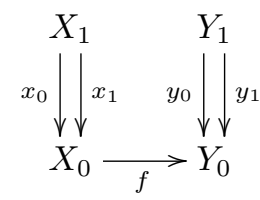

such that $x_{0} \cdot f \simeq x_{1} \cdot f$;

- a morphism in $\mathbb{C}_{r c}$ is an equivalence class $[f]$ of premorphisms : we identify two premorphisms $f$ and $g$ if $f \simeq g$;

- composition and identities in $\mathbb{C}_{r c}$, and the full embedding $\Lambda$, are the obvious ones.

Recall that a factorization system $(\mathcal{E}, \mathcal{M})$ on a category $\mathbb{B}$ (see $[\mathbf{2}]$ for the notion of factorization system) is proper if each $\mathcal{E}$-arrow is an epi and each $\mathcal{M}$-arrow is a mono ; it is stable if $\mathcal{E}$ is stable under pullbacks. A pre-regular category is a pair $\mathbb{B}=(\mathbb{B},(\mathcal{E}, \mathcal{M}))$ with $\mathbb{B}$ a category and $(\mathcal{E}, \mathcal{M})$ a proper and stable factorization system on $\mathbb{B}$. Observe that any regular category is pre-regular in a canonical way : take as $\mathcal{E}$ regular epis and as $\mathcal{M}$ monos. The pre-regular completion of a category has been introduced, in the special case of the stable homotopy category, by Freyd in [8]. It has been studied in all its generality in $[\mathbf{9}]$ (see also $[\mathbf{1 0}]$ ). We recall from [9] the following facts.

Proposition 3. Let $\mathbb{C}$ be a category. Then

1) there exists a pre-regular category $\mathbb{C}_{\text {preg }}$ and a full and faithful functor

$$
\Psi: \mathbb{C} \rightarrow \mathbb{C}_{\text {preg }}
$$

such that, for each pre-regular category $\mathbb{B}$, the composition with $\Psi$ induces an equivalence between the category of functors from $\mathbb{C}_{\text {preg }}$ to $\mathbb{B}$ preserving the factorization system and the category of functors from $\mathbb{C}$ to $\mathbb{B}$

$$
\Psi \cdot-: f s\left[\mathbb{C}_{\text {preg }}, \mathbb{B}\right] \rightarrow[\mathbb{C}, \mathbb{B}] ;
$$

2) for each object $C$ of $\mathbb{C}, \Psi(C)$ is $\mathcal{E}$-projective and $\mathcal{M}$-injective in $\mathbb{C}_{\text {preg. }}$. Moreover, for each object $X$ of $\mathbb{C}_{\text {preg }}$, there are objects $X_{0}, X^{\prime}$ in $\mathbb{C}$ and arrows $x_{0}: \Psi\left(X_{0}\right) \rightarrow X, x^{\prime}: X \rightarrow \Psi\left(X^{\prime}\right)$ with $x_{0}$ in $\mathcal{E}$ and $x^{\prime}$ in $\mathcal{M}$. As a consequence, in $\mathbb{C}_{\text {preg }} \mathcal{E}$-projectives coincide with $\mathcal{M}$-injectives and they are exactly the retracts of the objects $\Psi(C)$, for $C \in \mathbb{C}$.

The pre-regular category $\mathbb{C}_{\text {preg }}$ can be described in the following way:

- an object of $\mathbb{C}_{\text {preg }}$ is a morphism $x: X_{0} \rightarrow X^{\prime}$ in $\mathbb{C}$;

- a premorphism in $\mathbb{C}_{\text {preg }}$ is a pair $\left(f_{0}, f^{\prime}\right)$ of arrows in $\mathbb{C}$ making commutative 
the following diagram

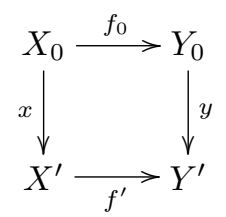

- a morphism in $\mathbb{C}_{\text {preg }}$ is an equivalence class $\left[f_{0}, f^{\prime}\right]$ of premorphisms : we identify two pairs $\left(f_{0}, f^{\prime}\right)$ and $\left(g_{0}, g^{\prime}\right)$ of premorphisms if $x \cdot f^{\prime}=x \cdot g^{\prime}$ (equivalently, if $\left.f_{0} \cdot y=g_{0} \cdot y\right)$;

- composition and identities in $\mathbb{C}_{\text {preg }}$, and the full embedding $\Psi$, are the obvious ones.

\section{Representations in abelian categories}

Since many of the interesting examples are given by locally small, but not small, categories, in this section we give elementary proofs, i.e. without using embedding in presheaf categories; all the facts quoted in the first section can be proved in this way. This allows us to remove size conditions from Freyd's results.

The second of the Freyd's results quoted in the introduction immediately follows from Proposition 1. (For the notions of preadditive, additive and abelian category we follow [2]. In particular, we will use that a category is abelian iff it is exact and (pre)additive.)

Proposition 4. [7] A category $\mathbb{C}$ is equivalent to a projective cover of an abelian category if and only if it is preadditive and it has weak finite products and weak kernels.

Proof: If $\mathbb{C}$ is a projective cover of an abelian category $\mathbb{A}$, then $\mathbb{C}$ is preadditive because $\mathbb{A}$ is preadditive. Moreover, given a finite diagram in $\mathbb{C}$, any $\mathbb{C}$-cover of the corresponding limit in $\mathbb{A}$ is a weak limit in $\mathbb{C}$.

Conversely, if $\mathbb{C}$ has weak kernels, then it has weak equalizers and then, if it has also weak finite products, it has all weak finite limits [6]. Now consider its exact comletion $\mathbb{C}_{e x}$ : since $\mathbb{C}$ is preadditive, also $\mathbb{C}_{e x}$ is preadditive (define $[f, \bar{f}]+[g, \bar{g}]=$ $[f+g, \bar{f}+\bar{g}])$ and then it is abelian. By point 2 of Proposition 1 , the proof is complete.

To go further in our analysis of Freyd's results, we now compare the exact completion and the reflexive coequalizer completion. The next lemma generalizes a result in $[\mathbf{1 2}]$ by deleting assumptions on sums.

Lemma 5. Let $\mathbb{C}$ be a category with weak finite limits. Then $\mathbb{C}_{e x}$ is equivalent to a full subcategory of $\mathbb{C}_{r c}$. 
Proof: Consider the functor $L: \mathbb{C}_{e x} \rightarrow \mathbb{C}_{r c}$

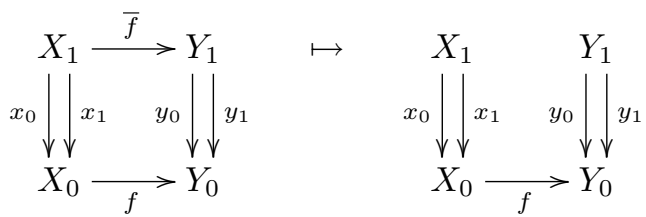

It is well-defined, in fact the arrow $\bar{f}$ gives that $f$ is a premorphism in $\mathbb{C}_{r c}$; moreover, if $[f, \bar{f}]=[g, \bar{g}]$ in $\mathbb{C}_{e x}$, then the arrow $l: X_{0} \rightarrow Y_{1}$ gives that $f$ and $g$ are preequivalent, and then $[f]=[g]$ in $\mathbb{C}_{r c}$.

Now observe that if $Y_{1} \underset{y_{1}}{\stackrel{y_{0}}{\longrightarrow}} \gtrless Y_{0}$ is a pseudo-equivalence in $\mathbb{C}$, then for each object $V$ the relation to be preequivalent in $\mathbb{C}\left(V, Y_{0}\right)$ is already an equivalence relation, so that we can avoid the passage to the generated equivalence relation. This implies that $[f, \bar{f}]=[g, \bar{g}]$ in $\mathbb{C}_{e x}$ if and only if $[f]=[g]$ in $\mathbb{C}_{r c}$, so that the functor $L$ is faithful. It is also full because, given a premorphism in $\mathbb{C}_{r c}$

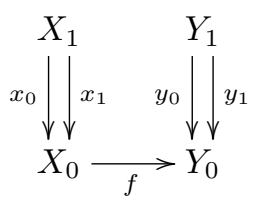

with $\left(y_{0}, y_{1}\right)$ a pseudo-equivalence, $x_{0} \cdot f \simeq x_{1} \cdot f$ means that they are preequivalent, so that there exists $\bar{f}: X_{1} \rightarrow Y_{1}$ such that $(f, \bar{f})$ is a premorphism in $\mathbb{C}_{e x}$.

Recall that a category with finite limits is a Mal'cev category if any reflexive relation is an equivalence relation $[4,5]$. Now we a little bit adapt this definition to the case of categories with weak finite limits, where it is more appropriate to work with graphs instead of relations.

Definition 6. Let $\mathbb{C}$ be a category with weak finite limits. We say that $\mathbb{C}$ is a $G$ Mal'cev category ( $G$ stays for graph) if any reflexive graph can be equipped with a structure of pseudo-equivalence.

Remark : Observe that, for a category $\mathbb{C}$ with weak finite limits, to be G-Mal'cev is equivalent to the fact that any representable functor $\mathbb{C}(C,-)$ sends reflexive graphs to pseudo-equivalences ; this is also equivalent to the fact that for each object $C$ and for each reflexive graph $X_{1} \longrightarrow X_{0}$, the jointly monic part of its (epi, jointly mono) factorization in $S E T$ is an equivalence relation

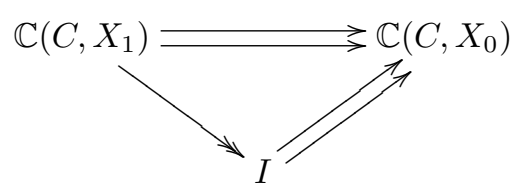

Observe also that any G-Mal'cev category having finite limits is Mal'cev.

Lemma 7. Let $\mathbb{C}$ be a G-Mal'cev category with weak finite limits. Then $\mathbb{C}_{e x}$ and $\mathbb{C}_{r c}$ are equivalent. 
Proof: If $\mathbb{C}$ is G-Mal'cev, then the functor $L: \mathbb{C}_{e x} \rightarrow \mathbb{C}_{r c}$ is surjective on objects and then, by Lemma 5 , it is an equivalence.

Lemma 8. Let $\mathbb{C}$ be a projective cover of a regular category $\mathbb{A}$. Then $\mathbb{A}$ is Mal'cev if and only if $\mathbb{C}$ is $G$-Mal'cev. In particular, a category $\mathbb{C}$ with weak finite limits is $G$-Mal'cev if and only if its exact completion $\mathbb{C}_{e x}$ is Mal'cev.

Proof: (only if) : Consider a reflexive graph $X_{1} \underset{x_{1}}{\stackrel{x_{0}}{\longrightarrow}} X_{0}$ in $\mathbb{C}$ and its (regular epi, jointly mono) factorization in $\mathbb{A}$

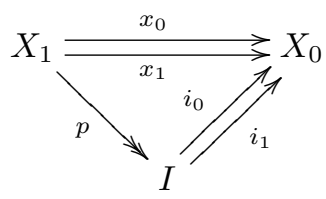

The relation $\left(i_{0}, i_{1}\right)$ is reflexive so that, by assumption, it is an equivalence relation. - Consider the symmetry $s: I \rightarrow I$ of $\left(i_{0}, i_{1}\right)$; since $X_{1}$ is regular projective and $p: X_{1} \rightarrow I$ is a regular epi, there exists a morphism $\sigma: X_{1} \rightarrow X_{1}$ such that $\sigma \cdot p=p \cdot s$; one checks that $\sigma$ is a symmetry for the graph $\left(x_{0}, x_{1}\right)$.

- Consider the following pullbacks in $\mathbb{A}$

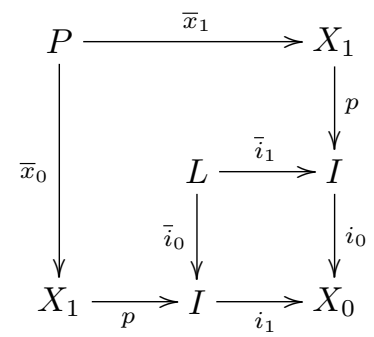

together with the unique factorization $\pi: P \rightarrow L$; consider now a regular epi $q: X_{2} \rightarrow P$ with $X_{2}$ in $\mathbb{C}$. Then

$$
X_{1} \stackrel{q \cdot \bar{x}_{0}}{\longleftarrow} X_{2} \stackrel{q \cdot \bar{x}_{1}}{\longrightarrow} X_{1}
$$

is a weak pullback of $x_{0}$ and $x_{1}$ in $\mathbb{C}$. Let $t: L \rightarrow I$ be given by the transitivity of $\left(i_{0}, i_{1}\right)$; since $X_{2}$ is regular projective and $p: X_{1} \rightarrow I$ is a regular epi, there exists a morphism $\tau: X_{2} \rightarrow X_{1}$ such that $\tau \cdot p=q \cdot \pi \cdot t$; one checks that $\tau$ yields transitivity of $\left(x_{0}, x_{1}\right)$.

(if) : Consider a reflexive relation $R \underset{r_{1}}{\stackrel{r_{0}}{\longrightarrow} X}$ in $\mathbb{A}$, with $\delta: X \rightarrow R$ the common section of $r_{0}$ and $r_{1}$, an object $\bar{X}$ in $\mathbb{C}$ and a regular epi $x: \bar{X} \rightarrow X$. Consider also 
the following pullback

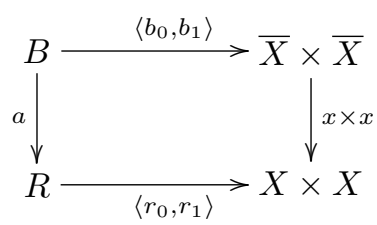

and cover $B$ with a regular epi $\beta: P \rightarrow B$ from an object $P$ of $\mathbb{C}$. Since $x \cdot \delta \cdot\left\langle r_{0}, r_{1}\right\rangle=$ $\Delta \cdot(x \times x)$, where $\Delta: \bar{X} \rightarrow \bar{X} \times \bar{X}$ is the diagonal, there is a unique $\alpha: \bar{X} \rightarrow B$ such that $\alpha \cdot a=x \cdot \delta$ and $\alpha \cdot\left\langle b_{0}, b_{1}\right\rangle=\Delta$. Since $\beta$ is a regular epi and $\bar{X}$ is regular projective, there exists $\gamma: \bar{X} \rightarrow P$ such that $\gamma \cdot \beta=\alpha$. Now $\gamma \cdot \beta$ is a reflection for the graph $P \underset{\beta \cdot b_{1}}{\stackrel{\beta \cdot b_{0}}{\longrightarrow}} \bar{X}$ in $\mathbb{C}$. By assumption, this graph has a structure of pseudoequivalence. Let $\sigma: P \rightarrow P$ be a symmetry. Then $\sigma$ is a symmetry also for the graph $P \underset{\beta \cdot b_{1} \cdot x}{\stackrel{\beta \cdot b_{0} \cdot x}{\longrightarrow}} X$ and then $R \underset{r_{1}}{\stackrel{r_{0}}{\longrightarrow}} X$, being the jointly monic part of its factorization, is a symmetric relation. Since $\mathbb{A}$ is regular, this is enough to prove that it is a Mal'cev category.

Recall from [2] that a preadditive category is additive iff it has finite sums, and that a functor between additive categories is additive iff it preserves finite sums. Moreover, for a functor between abelian categories, to be right exact or exact in the sense of abelian categories is the same as to be right exact or exact in the sense used in section 1.

Proposition 9. Let $\mathbb{C}$ be a preadditive category with weak finite products and weak kernels. Then there exists an abelian category $\mathbb{C}_{a b}$ and a full and faithful functor

$$
\Sigma: \mathbb{C} \rightarrow \mathbb{C}_{a b}
$$

such that, for each abelian category $\mathbb{B}$, the composition with $\Sigma$ induces an equivalence between the category of right exact functors from $\mathbb{C}_{a b}$ to $\mathbb{B}$ and the category of additive functors from $\mathbb{C}$ to $\mathbb{B}$

$$
\Sigma \cdot-: \operatorname{Right}\left[\mathbb{C}_{a b}, \mathbb{B}\right] \rightarrow \operatorname{Add}[\mathbb{C}, \mathbb{B}]
$$

Proof: We already know that such a category $\mathbb{C}$ has weak finite limits. Moreover, up to replacing $\mathbb{C}$ by its Cauchy-completion $\overline{\mathbb{C}}$, which is nothing else but the full subcategory of all the regular projective objects of the abelian category $\mathbb{C}_{e x}$, we can assume that $\mathbb{C}$ has finite sums. (In fact $\overline{\mathbb{C}}$ is still preadditive, and a functor $\mathbb{C} \rightarrow \mathbb{B}$ is additive iff its unique extension $\overline{\mathbb{C}} \rightarrow \mathbb{B}$ is additive.) Now we can take as $\mathbb{C}_{a b}$ the exact completion $\mathbb{C}_{e x}$. It is abelian and then it is a Mal'cev category $[\mathbf{4}, \mathbf{5}]$. By Lemma 7 and Lemma 8, it follows that $L: \mathbb{C}_{e x} \rightarrow \mathbb{C}_{r c}$ is an equivalence. Now take as $\Sigma: \mathbb{C} \rightarrow \mathbb{C}_{a b}$ the functor $\Lambda \cdot L^{-1}: \mathbb{C} \rightarrow \mathbb{C}_{r c} \rightarrow \mathbb{C}_{e x}$; point 2 of Proposition 2 gives us exactly the requested universal property.

Bearing in mind point 3 of Proposition 1, we have the following corollary, which is the first of Freyd's results quoted in the introduction. 
Corollary 10. [7] Let $\mathbb{A}$ be an abelian category and $\mathbb{P}$ a projective cover of $\mathbb{A}$. Then, for each abelian category $\mathbb{B}$, the composition with the full inclusion $\mathbb{P} \rightarrow \mathbb{A}$ induces an equivalence of categories Right $[\mathbb{A}, \mathbb{B}] \rightarrow \operatorname{Add}[\mathbb{P}, \mathbb{B}]$.

\section{Frobenius categories}

To explain the universal property of a Frobenius category (Theorem 3.1 in [8]), in this section we compare the exact and the pre-regular completion of a category.

Let $\mathbb{C}$ be a category with weak finite limits. There is a canonical (pseudo) functor $K: \mathbb{C}_{\text {preg }} \rightarrow \mathbb{C}_{e x}$ induced by the universal property of $\mathbb{C}_{\text {preg }}$. Explicitely, $K$ is defined in the following way

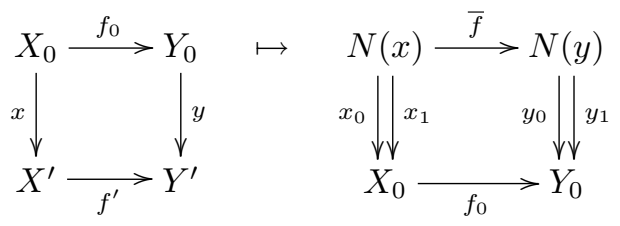

where $\left(x_{0}, x_{1}\right)$ is a weak kernel pair of $x$ and $\bar{f}$ is induced by the fact that $\left(y_{0}, y_{1}\right)$ is a weak kernel pair of $y$. The functor $K$ is well defined because two weak kernel pairs of the same arrow are objects canonically isomorphic in $\mathbb{C}_{e x}$. Moreover, $K$ is faithful.

Lemma 11. Let $\mathbb{C}$ be a category with weak finite limits. The functor $K: \mathbb{C}_{\text {preg }} \rightarrow$ $\mathbb{C}_{e x}$ is an equivalence if and only if the following conditions hold in $\mathbb{C}$ :

$i$ - each arrow is a weak coequalizer ;

ii - each pseudo-equivalence is a weak kernel pair.

Proof: (if) : Consider two objects $x: X_{0} \rightarrow X^{\prime}$ and $y: Y_{0} \rightarrow Y^{\prime}$ in $\mathbb{C}_{\text {preg }}$ and an arrow $\left[f_{0}, \bar{f}\right]: K(x) \rightarrow K(y)$ in $\mathbb{C}_{e x}$

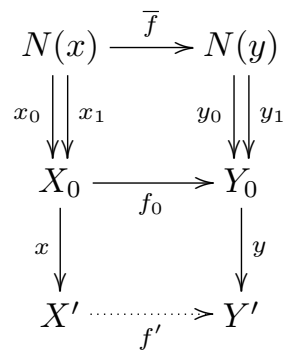

Since $x$ is, by condition (i), a weak coequalizer, it is a weak coequalizer of $\left(x_{0}, x_{1}\right)$. Then there exists $f^{\prime}$ such that $x \cdot f^{\prime}=f_{0} \cdot y$, so that $K$ is full.

Now consider an object $C_{1} \underset{r_{1}}{\stackrel{r_{0}}{\longrightarrow}} C_{0}$ in $\mathbb{C}_{e x}$. By condition (ii), $\left(r_{0}, r_{1}\right)$ is a weak kernel pair of some $q: C_{0} \rightarrow Q$ and then $\left(r_{0}, r_{1}\right)$ and $K(q)$ are isomorphic. 
(only if) : Consider the following diagram

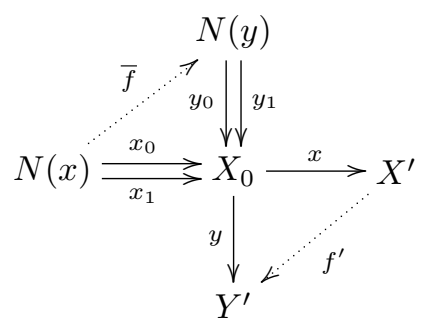

where $x$ is an arrow in $\mathbb{C},\left(x_{0}, x_{1}\right)$ is a weak kernel pair of $x, y$ coequalizes $x_{0}$ and $x_{1}$ and $\left(y_{0}, y_{1}\right)$ is a weak kernel pair of $y$. The condition $x_{0} \cdot y=x_{1} \cdot y$ implies that there exists $\bar{f}$ such that $\bar{f} \cdot y_{0}=x_{0}$ and $\bar{f} \cdot y_{1}=x_{1}$. Now $\left[1_{X^{\prime}}, \bar{f}\right]$ is an arrow in $\mathbb{C}_{e x}$ between $K(x)$ and $K(y)$. Since $K$ is full, there is an arrow $f^{\prime}$ such that $x \cdot f^{\prime}=y$. This means that $x$ is a weak coequalizer of $\left(x_{0}, x_{1}\right)$.

To prove condition (ii), we identify $\mathbb{C}$ with a full subcategory of $\mathbb{C}_{e x}$. Let $C_{1} \underset{r_{1}}{\stackrel{r_{0}}{\rightleftarrows}} C_{0}$ be a pseudo-equivalence in $\mathbb{C}$, and consider the following diagram in $\mathbb{C}_{e x}$

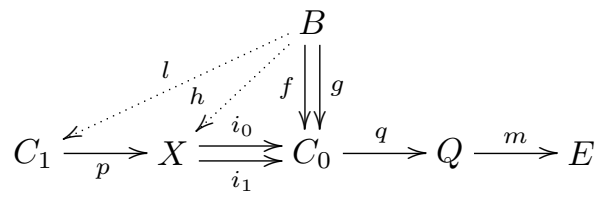

where $\left(p,\left(i_{0}, i_{1}\right)\right)$ is the regular epi-jointly monic factorization of $\left(r_{0}, r_{1}\right)$ and $q$ is the coequalizer of $\left(i_{0}, i_{1}\right)$, which exists by Theorem 26 in [6]. Moreover, since $K$ is an equivalence, there is an object $E$ in $\mathbb{C}$ and a mono $m: Q \rightarrow E$. Now we prove that $\left(r_{0}, r_{1}\right)$ is a weak kernel pair in $\mathbb{C}$ of $q \cdot m$. Assume that $f \cdot q \cdot m=g \cdot q \cdot m$. Since $m$ is a mono and $\left(i_{0}, i_{1}\right)$ is the kernel pair of $q$, there exists a unique $h$ such that $h \cdot i_{0}=f$ and $h \cdot i_{1}=g$. Since $B$ is regular projective and $p$ is a regular epi, there is $l$ such that $l \cdot p=h$. Finally, $l \cdot r_{0}=f$ and $l \cdot r_{1}=g$.

Remark : The previous lemma can be made more precise. The functor $K: \mathbb{C}_{\text {preg }} \rightarrow$ $\mathbb{C}_{e x}$ factors through $\mathbb{C}_{r e g}$, the regular completion of $\mathbb{C}[\mathbf{6}]$, and one can prove that $\mathrm{i}-\mathbb{C}_{\text {preg }} \rightarrow \mathbb{C}_{\text {reg }}$ is full iff in $\mathbb{C}$ any arrow is a weak coequalizer ;

ii - $\mathbb{C}_{r e g} \rightarrow \mathbb{C}_{e x}$ is an equivalence iff for any pseudo-equivalence $C_{1} \underset{r_{1}}{\stackrel{r_{0}}{\longrightarrow}} C_{0}$ in $\mathbb{C}$ there exists a finite family of arrows $\left(q_{i}: C_{0} \rightarrow Q_{i}\right)_{I}$ such that $\left(r_{0}, r_{1}\right)$ is weak universal w.r.t. the condition $r_{0} \cdot q_{i}=r_{1} \cdot q_{i}$ for all $i$ in $I$.

Recall that a Frobenius category is an abelian category with enough projectives and injectives, and in which projectives and injectives coincide. In the additive case, the conditions of the previous lemma are equivalent to the fact that $\mathbb{C}$ is a projective-injective cover of a Frobenius category. In fact, we have the following lemma (compare with Theorem 1.7 in [7]).

Lemma 12. Let $\mathbb{C}$ be an additive category with weak kernels, and assume that in $\mathbb{C}$ each arrow is a weak cokernel. The following conditions are equivalent: 
1) each arrow has a weak cokernel, each arrow is a weak kernel ;

2) each pseudo-equivalence is a weak kernel pair.

Proof: 1$) \Rightarrow 2$ ) : Let $C_{1} \underset{r_{1}}{\stackrel{r_{0}}{\longrightarrow}} C_{0}$ be a pseudo-equivalence in $\mathbb{C}$ and consider a weak cokernel $q: C_{0} \rightarrow Q$ of $r_{0}-r_{1}$. Now we prove that $\left(\begin{array}{c}q \\ -q\end{array}\right): C_{0} \oplus C_{0} \rightarrow Q$ is a weak cokernel of $\left\langle r_{0}, r_{1}\right\rangle: C_{1} \rightarrow C_{0} \oplus C_{0}$. For this, consider an arrow $\left(\begin{array}{c}h_{1} \\ h_{2}\end{array}\right): C_{0} \oplus$ $C_{0} \rightarrow Z$ such that $\left\langle r_{0}, r_{1}\right\rangle \cdot\left(\begin{array}{c}h_{1} \\ h_{2}\end{array}\right)=0$ and let $i: C_{0} \rightarrow C_{1}$ be such that $i \cdot r_{0}=$ $1_{C_{0}}=i \cdot r_{1}$. We have $0=i \cdot 0=i \cdot\left(r_{0} \cdot h_{1}+r_{1} \cdot h_{2}\right)=i \cdot r_{0} \cdot h_{1}+i \cdot r_{1} \cdot h_{2}=h_{1}+h_{2}$, so that $h_{2}=-h_{1}$ and then $0=r_{0} \cdot h_{1}+r_{1} \cdot h_{2}=\left(r_{0}-r_{1}\right) \cdot h_{1}$. But $q$ is a weak cokernel of $r_{0}-r_{1}$, so that there exists $z: Q \rightarrow Z$ such that $q \cdot z=h_{1}$. This implies that $\left(\begin{array}{c}q \\ -q\end{array}\right) \cdot z=\left(\begin{array}{c}h_{1} \\ h_{2}\end{array}\right)$, that is $\left(\begin{array}{c}q \\ -q\end{array}\right)$ is a weak cokernel of $\left\langle r_{0}, r_{1}\right\rangle$. By assumption, $\left\langle r_{0}, r_{1}\right\rangle$ is a weak kernel, and then it is a weak kernel of its weak cokernel $\left(\begin{array}{c}q \\ -q\end{array}\right)$. This implies that $C_{1} \underset{r_{1}}{\stackrel{r_{0}}{\longrightarrow}} C_{0}$ is a weak kernel pair of $q$. In fact, if $T \underset{y}{\stackrel{x}{\longrightarrow}} C_{0}$ are such that $x \cdot q=y \cdot q$, then $\langle x, y\rangle \cdot\left(\begin{array}{c}q \\ -q\end{array}\right)=0$ and then there is $t: T \rightarrow C_{1}$ such that $t \cdot\left\langle r_{0}, r_{1}\right\rangle=\langle x, y\rangle$. This means $t \cdot r_{0}=x$ and $t \cdot r_{1}=y$. $2) \Rightarrow 1$ ) : Let $f: X \rightarrow Y$ be an arrow in $\mathbb{C}$ (which we identify with a full subcategory of the abelian category $\mathbb{C}_{e x}$ ). Consider the (regular epi, mono) factorization of $f$ in $\mathbb{C}_{e x}$ and the cokernel $q$ of its monic part $i$

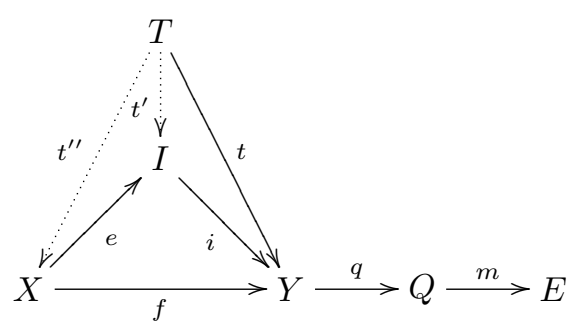

and embed $Q$ in an object $E$ of $\mathbb{C}$ (this is possible because $\mathbb{C}$ has weak finite limits and satisfies the conditions of the previous lemma, so that $K: \mathbb{C}_{\text {preg }} \rightarrow \mathbb{C}_{\text {ex }}$ is an equivalence). Let us show that $f$ is a weak kernel of $q \cdot m$ in $\mathbb{C}$. Let $t: T \rightarrow Y$ be an arrow in $\mathbb{C}$ such that $t \cdot q \cdot m=0$. Since $m$ is a mono, then $t \cdot q=0$. But $i$ is a mono, so that it is the kernel of its cokernel $q$, and then there exists a unique $t^{\prime}$ such that $t^{\prime} \cdot i=t$. Since $T$ is regular projective and $e$ is a regular epi, there is $t^{\prime \prime}$ such that $t^{\prime \prime} \cdot e=t^{\prime}$. Finally, $t^{\prime \prime} \cdot f=t$, so that $f$ is a weak kernel of $q \cdot m$. Moreover, by assumption, $q \cdot m$ is a weak cokernel, so that it is a weak cokernel of its weak kernel $f$.

Corollary 13. [8] Let $\mathbb{C}$ be an additive category such that each arrow has a weak kernel and a weak cokernel, and it is a weak kernel and a weak cokernel. Then $\mathbb{C}_{\text {preg }}$ 
is a Frobenius category. Moreover, for any abelian category $\mathbb{B}$ and for any additive functor $F: \mathbb{C} \rightarrow \mathbb{B}$, there are three functors $F_{i}: \mathbb{C}_{\text {preg }} \rightarrow \mathbb{B}(i=1,2,3)$ such that :

1) The following diagram commutes, up to isomorphism, for $i=1,2,3$

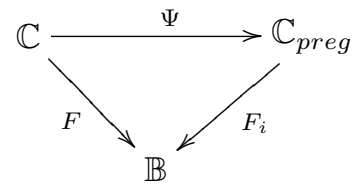

2) $F_{1}$ is right exact, $F_{2}$ is left exact and $F_{3}$ preserves images ;

3) Each $F_{i}$ is essentially unique within these properties.

Proof: By Lemmas 7, 8, 11 and 12, we have two equivalences

$$
K: \mathbb{C}_{\text {preg }} \rightarrow \mathbb{C}_{e x} \quad L: \mathbb{C}_{e x} \rightarrow \mathbb{C}_{r c}
$$

Since $\mathbb{C}$ is additive, $\mathbb{C}_{e x} \simeq \mathbb{C}_{\text {preg }}$ is abelian. It is Frobenius by point 2 of Proposition 3. Now, the existence of $F_{3}$ follows from the universal property of $\mathbb{C}_{\text {preg }}$ because, under the equivalence $\mathbb{C}_{\text {preg }} \simeq \mathbb{C}_{e x}$, the factorization system in $\mathbb{C}_{\text {preg }}$ becomes the (regular epi, mono) factorization. As far as $F_{1}$ is concerned, we simply apply Proposition 9 using the equivalence $\mathbb{C}_{\text {preg }} \simeq \mathbb{C}_{r c}$. Finally, since $\mathbb{C}_{\text {preg }}$ is abelian, its dual category is exact. Moreover, by point 2 of Proposition $3, \mathbb{C}^{o p}$ is a projective cover of $\left(\mathbb{C}_{\text {preg }}\right)^{o p}$. We obtain now $F_{2}$ applying Proposition 9 to the dual categories.

To end this paper, we consider the case of triangulated categories. For definitions and basic facts, the reader can consult $[\mathbf{1 1}, \mathbf{1 4}]$. The following lemma shows that the notion of homological functor on a triangulated category, which is defined in terms of the triangulated structure, is in fact an intrinsic notion.

Lemma 14. Let $\mathbb{T}$ be a triangulated category and $\mathbb{B}$ an abelian category. An additive functor $H: \mathbb{T} \rightarrow \mathbb{B}$ is a homological functor if and only if it is left covering.

Proof: Since $H$ is additive, we have to prove that $H$ is left covering w.r.t. weak kernels iff it sends triangles on exact sequences. This is the case because any arrow $v: Y \rightarrow Z$ fits into an triangle of the form $(u, v, w)$ and, in any triangle

$$
X \stackrel{u}{\longrightarrow} Y \stackrel{v}{\longrightarrow} Z \stackrel{w}{\longrightarrow} \Sigma X,
$$

$u: X \rightarrow Y$ is a weak kernel of $v$. Now the exactness of

$$
H(X) \stackrel{H(u)}{\longrightarrow} H(Y) \stackrel{H(v)}{\longrightarrow} H(Z)
$$

means that the comparison between $H(u)$ and the kernel of $H(v)$ is a (regular) epi, i.e. that $H$ is left covering w.r.t. weak kernels.

Corollary 15. Let $\mathbb{T}$ be a triangulated category. Then $\Gamma: \mathbb{T} \rightarrow \mathbb{T}_{e x}$ is the universal homological functor on $\mathbb{T}$.

Proof: Since $\mathbb{T}$ is additive, $\mathbb{T}_{e x}$ is abelian. The statement follows now from Lemma 14 and point 1 of Proposition 1. 
Since any triangulated category satisfies the conditions of Lemma 12, in the previous corollary we can replace the exact completion by the pre-regular completion. We get in this way the theorem proved in [8] for the stable homotopy category, and in $[\mathbf{9}, \mathbf{1 1}]$ for an arbitrary triangulated category.

\section{References}

[1] M. Barr. Exact Categories, Springer LNM 236 (1971) 1-120.

[2] F. Borceux. Handbook of Categorical Algebra, Cambridge University Press (1994).

[3] M. Bunge, A. Carboni. The symmetric topos, J. Pure Appl. Algebra 105 (1995) 233-249.

[4] A. Carboni, G.M. Kelly, M.C. Pedicchio. Some remarks on Mal'cev and Goursat categories, Appl. Categ. Structures 1 (1993) 385-421.

[5] A. Carboni, J. Lambek, M.C. Pedicchio. Diagram chasing in Mal'cev categories, J. Pure Appl. Algebra 69 (1990) 271-284.

[6] A. Carboni, E.M. Vitale. Regular and exact completions, J. Pure Appl. Algebra 125 (1998) 79-116.

[7] P. Freyd. Representations in Abelian categories, Proc. Conf. Categor. Algebra La Jolla 1965 (1966) 95-120.

[8] P. Freyd. Stable homotopy, Proc. Conf. Categor. Algebra La Jolla 1965 (1966) 121-172.

[9] M. Grandis. Weak subobjects and the epi-monic completion of a category, J. Pure Appl. Algebra 154 (2000) 193-212.

[10] S. Lack, E.M. Vitale. When do completion processes give rise to extensive categories? J. Pure Appl. Algebra 159 (2001) 203-230.

[11] A. Neeman. Triangulated categories, Princeton University Press (2001).

[12] M.C. Pedicchio, J. Rosický. Comparing coequalizer and exact completions, Theory and Applications of Categories 6 (1999) 77-82.

[13] A. Pitts. The lex reflection of a category with finite products, unpublished notes (1996).

[14] C.A. Weibel. An introduction to homological algebra, Cambridge University Press (1994). 
This article may be accessed via WWW at http://www.rmi.acnet.ge/hha/ or by anonymous ftp at

ftp://ftp.rmi.acnet.ge/pub/hha/volumes/2001/n3a1/v3n3a1.(dvi,ps,pdf)

J. Rosický rosicky@math.muni.cz

Department of Mathematics

Masaryk University

Janáčkovo nám. 2a, 66295 Brno

Czech Republic

E.M. Vitale vitale@agel.ucl.ac.be

Département de Mathématique

Université catholique de Louvain

Chemin du Cyclotron 2, 1348 Louvain-la-Neuve

Belgium 